\title{
Elevated serum neopterin is associated with increased risk of cardiovascular events in acute coronary syndromes
}

\author{
Anwar Santoso ${ }^{1}$, Sri Wardani ${ }^{2}$, and Ketut Surayana ${ }^{3}$
}

B

ACKGROUND: Neopterin is a soluble biomarker of monocyte activation and its increased concentration might be expressed in atherosclerosis. Until recently, there has been lacking of information on the prognostic role of neopterin in acute coronary syndromes (ACS). The study was aimed at measuring the associations between elevated serum neopterin and increased risk of cardiovascular (CV) events in ACS.

METHODS AND RESULTS: This was a prospective cohort study, recruited 71 ACS patients from January 31 through August 31, 2007 in Sanglah Hospital of Udayana School of Medicine, Denpasar - Bali. Cardiovascular events, such as: $\mathrm{CV}$ death, recurrent myocardial infarction, stroke and recurrent myocardial ischemia were previously defined. Relative risk and survival rate were measured successively by Cox proportional model and Kaplan-Meier curve.

Of 71 ACS patients aged $56.8 \pm 9.5$ years, $21(29.5 \%)$ subjects underwent CV events. Overall mean followup was 151.6 (95\% CI: 129.7 - 173.5) days. Baseline characteristic were similarly distributed between groups with the highest quartile neopterin level $(\geq 14.7$ $\mathrm{nmol} / \mathrm{L})$ than those with lowest quartile $(\leq 6.2 \mathrm{nmol} /$ L). Patients with the highest quartile had the worst survival curve than those with the lowest quartile (log-rank test; $\mathrm{P}=0.047$ ). On Cox proportional model, relative risk of highest quartile group was 5.84 (95\% CI: 1.19 -28.47; $\mathrm{P}=0.029$ ) compared to lowest quartile, after being adjusted with other predictors.

CONCLUSIONS: Elevated serum neopterin is associated with increased risk of $\mathrm{CV}$ events in acute coronary syndromes.
KEYWORDS: neopterin, cardiovascular events, acute coronary syndromes.

\section{Introduction}

Inflammation plays an important role in atherosclerosis and plaque vulnerability (1). Activated macrophages contribute to both atherosclerotic plaque disruption and intracoronary thrombus formation, often leading to acute coronary syndromes (ACS). Plaque disruption usually takes place at the thinnest fibrous cap and is mostly infiltrated with macrophages (2). The activated macrophages produce inflammatory cytokines, protease, and cytotoxic oxygen and nitrogen radical molecules (3). Macrophages activated by interferon- $\gamma$ $(\mathrm{IFN}-\gamma)$ also synthesize neopterin (4).

Neopterin, a pteridine derivative and a byproduct of the guanosine triphosphate-biopterin pathway and produced by activated monocyte $(5,6)$ is thought to represent a marker of immune activation and macrophage activity (4). It is elevated in a number of autoimmune and infectious disease states (6). Because of its relative stability and ease of assay has made it a subject for research as a mean to monitor disease activity. Acute inflammatory markers such as high neutrophil count, hs-CRP, and neopterin concentration correlate with the presence of multiple angiographically complex coronary stenoses. Neopterin concentration was considered as a stronger predictor of multiple complex plaques than were neutrophil count and hs-CRP (7).

A previous study has shown that serum neopterin is an independent predictor of major adverse coronary

\footnotetext{
${ }^{1}$ Department of Cardiology - Vascular Medicine, Faculty of Medicine - Udayana University, Denpasar

${ }^{2}$ Department of Internal Medicine $\sim$ Sanglah Hospital, Denpasar

${ }^{3}$ Department of Internal Medicine, Faculty of Medicine - Udayana University, Denpasar
} 
events in those with chronic stable angina pectoris (8). It is also elevated in patients with ACS compared to those with stable ischemic heart disease (9). Likewise, high serum neopterin levels predict the development of acute coronary events in women with chronic stable angina pectoris (10) and hypertensive patients without significant obstructive coronary artery disease (CAD) (11). Until recently, unfortunately, there has been lack of scientific information on the prognostic value of neopterin in ACS in non-Caucasian population. Only one study reported elevated plasma neopterin level, which might identify patients at long-term risk of death or recurrent acute coronary events after ACS in Caucasian (12).

In the present study we prospectively measured the associations between elevated serum neopterin and increased risk of cardiovascular (CV) events in ACS in Bali. Specifically, we assessed the relative risk of elevated serum neopterin in association with $\mathrm{CV}$ events and survival rate of patients with ACS.

\section{Patients \& Methods}

\section{PATIENTS AND STUDY PROTOCOLS}

This was a prospective cohort study carried out from January 31 to August 31, 2007, involving 77 ACS patients admitted to Sanglah Hospital of Udayana University School of Medicine, Denpasar-Bali. Patients with ACS (aged 30 - 74 years) were consecutively recruited in the study, who had agreed to participate by signing the written informed consent. The local ethics committee of our hospital approved the study. This is a top referral Public Hospital (teaching hospital as well) for Bali Island and its surrounding smaller islands.

Exclusion criteria included ACS subjects with rheumaticheart disease, acute and chronic liver disease, chronic kidney disease (represented by creatinin clearance using Cockroft Gault formula $\leq 60 \mathrm{ml} / 1.73$ $\mathrm{m} 2 / \mathrm{min}$ ), infectious disease, and malignancy.

\section{DEFINITIONS OF CARDIOVASCULAR RISK FACTORS}

Hypertension was diagnosed in the presence of a systolic blood pressure of $\geq 140 \mathrm{mmHg}$ and/or diastolic blood pressure of $\geq 90 \mathrm{mmHg}$ as measured on at least two separate occasions. Patients receiving antihypertensive medications were also regarded to suffer hypertension, irrespective of the blood pressure recordings at study entry (13). Patients were considered to have diabetes mellitus if they were taking active treatment with insulin or oral hypoglycemic agents, or the fasting blood glucose $\geq 126 \mathrm{mg} / \mathrm{dL}$ and/or random blood glucose $\geq 200 \mathrm{mg} / \mathrm{dL}$ (14). Hypercholesterolemia was defined as a documented total cholesterol concentration $>200 \mathrm{mg} / \mathrm{dL}$. Smoking status was defined as: a) non-smoker for those who never had smoked, b) ex-smoker, for those who ever had smoked but already had quit at study entry, c) current smoker, those which was active smoker at study entry (15). Obesity was diagnosed by measuring body mass index.

\section{BIOCHEMICAL MEASUREMENTS}

Venous blood was collected from all subjects at the time of admission for routine laboratory examination. Serum neopterin concentration was taken at 3rd hospitalization day, and measured using a commercially available immunoassay kit (ELISA Kit, IBL, Hamburg, Germany) by a qualified laboratory technician blinded to clinical characteristics and CV events. The within-coefficient variability was $3.6-6.8 \%$ in the 7.7 $-48 \mathrm{nmol} / \mathrm{L}$ range; it has very good correlation with HPLC (high performance liquid chromatography) measurement $(r=0.97)$. Limit of detection reached as low as $0.7 \mathrm{nmol} / \mathrm{L}$ (16). Neopterin concentrations were divided into quartile ranges with regard to planned analysis, as shown by previous study (12).

\section{CLINICAL CHARACTERIZATION, FOLLOW-UP, AND CLINICAL OUTCOMES}

Data acquired at study entry included age, gender, height, weight, blood pressure, risk factors history including diabetes mellitus, hypertension, and family history of CAD, smoking, dyslipidemia. Killip classification and the Canadian Cardiovascular Society (CCS) functional class, as well as cardiac medications were recorded at study entry.

After recruitment, routinebaselinecharacterization was carried out in all subjects, who were then followed for up to 7 months of study period. All subjects, after discharged from the hospital were encouraged to visit the outpatient clinic unit of Sanglah Hospital. Subjects who did not regularly visit the hospital were further followed up by performing home visit. This effort was made to avoid loss-to follow-up. At the end of study, a total of 71 ACS subjects remained in the analysis, and 
6 subjects had refused to participate.

The cardiovascular events determined as prespecified clinical outcomes for this analysis included $\mathrm{CV}$ mortality, recurrent myocardial infarction, stroke and recurrent myocardial ischemia. These clinical outcomes were assessed by research assistants.

\section{STATISTICAL ANALYSIS}

Prior to beginning of the study, we calculated the number of the patients needed to have a power of $80 \%$ with predicted relative risk of 3.7 at the 0.05 level of significance. The estimated minimal sample size was 70.

Normally distributed continuous variables were expressed as the mean \pm standard deviation (SD), and continuous variables with non-normal distribution were presented as median values (interquartile range). Analysis of normality of the continuous variables was performed with the Kolmogorov-Smirnov test. Homogeneity of variance was also tested using the Leven's test.

In order to have true association between elevated serum neopterin and clinical outcomes of interest, we constructed a Cox proportional model that adjusted for age, history of diabetes mellitus, hypertension, and family history of CAD to assess relative risk over 7 months. Relative risk was expressed with 95\% confidence interval. Survival rate was also determined using Kaplan-Meier survival curve. Differences were considered to be statistically significant if the null hypothesis would be rejected with $>95 \%$ confidence. The SPSS 13.0 statistical software package was used for calculations.

The authors had full access to the data and take full responsibility for its integrity. All authors have read and agree to the manuscript as written.

\section{Results}

\section{PARTICIPANTS}

Initially 77 subjects with ACS were recruited in the study, of which approximately $40 \%$ had an index diagnosis of ST-elevation myocardial infarction (MI), $13 \%$ had a diagnosis of non-ST elevation MI and the rest had unstable angina. Eventually, 71 subjects remained in the analysis and 6 subjects refused to participate without any reason. No loss to follow-up was noted in this study.

Neopterin concentrations were divided into quartile ranges. Elevated serum neopterin group was those subjects with neopterin concentration $\geq$ $14.72 \mathrm{nmol} / \mathrm{L}$. Along 213 median-days of follow-up, $21(28.2 \%)$ subjects underwent CV events. Baseline clinical characteristics, biochemical and treatment pattern results are shown in Table 1. All the clinical characteristics, biochemical and treatment pattern are identical between groups.

\section{CLINICAL OUTCOMES}

The cardiovascular events determined as prespecified clinical outcomes for this analysis included CV mortality, recurrent MI, stroke and recurrent myocardial ischemia, as presented in Table 2. Along 213 median-days of follow-up, $28.2 \%$ subjects underwent
CV events, namely: 4 (19.0\%) subjects had CV deaths, $2(9.5 \%)$ had recurrent MI and $15(71.5 \%)$ subjects suffered recurrent myocardial ischemia.

\section{KAPLAN-MEIER SURVIVAL CURVE}

Kaplan-Meier survival curve shows that the highest quartile group of neopterin had survival curve significantly worse than those in the lowest quartile group (log-rank test; $\mathrm{P}=0.047$ ), as presented in Figure 1. The highest quartile group had the mean survival shorter compared to the lowest quartile group $\{69.7$ (95\% CI: 48.1 - 91.2 days) vs. 190.5 (95\% CI: 161.1 219.8); $\mathrm{P}=0.047$ days $\}$.

\section{RISK OF CARDIOVASCULAR EVENTS}

After multivariable adjustment, the relative risk of $\mathrm{CV}$ events is significantly associated with a serum neopterin concentration of $\geq 14.72 \mathrm{nmol} / \mathrm{L}$ (RR, 5.84 [95\% CI: 1.19 to 28.47]; $\mathrm{P}=0.029$ ). Before being adjusted with other predictors, the RR was 6.63 (95\% CI: 1.37 to 32$) ; \mathrm{P}=0.018$. The other predictors being considered as important risk factors were age, history of hypertension, diabetes mellitus, and family history of CAD. 
Table 1. Baseline characteristics of 71 consecutive subjects with ACS

\begin{tabular}{|c|c|c|c|}
\hline & & Low neopterin group* & High neopterin group $†$ \\
\hline Age, (year) & & $56.4 \pm 9.9$ & $58.0 \pm 8.2$ \\
\hline Male gender, (\%) & & 71.7 & 77.8 \\
\hline \multicolumn{4}{|l|}{ Blood pressure, $\mathrm{mmHg}$} \\
\hline ystolic & & $120.2 \pm 17.7$ & $15.1 \pm 13.3$ \\
\hline iastolic & & $72.3 \pm 12.5$ & $72.2 \pm 12.4$ \\
\hline Body mass index, $\mathrm{kg} / \mathrm{m} 2$ & & $24.9 \pm 2.9$ & $25.5 \pm 3.0$ \\
\hline History of diabetes, (\%) & & 20.8 & 22.2 \\
\hline History of hypertension, (\%) & & 58.5 & 77.8 \\
\hline Family history of CAD‡, (\%) & & 37.7 & 44.6 \\
\hline \multicolumn{4}{|l|}{ Diagnosis } \\
\hline STEMI§, (\%) & & 39.6 & 44.4 \\
\hline Non-STEMI, (\%) & & 9.4 & 16.7 \\
\hline Unstable angina, (\%) & & 50.9 & 38.9 \\
\hline \multicolumn{4}{|l|}{ Laboratory } \\
\hline Leucocyte count & 9 & $.6 \pm 3.4$ & $9.5 \pm 2.7$ \\
\hline lood sugar, mg/dL & & $147.4 \pm 63.0$ & $128.9 \pm 49.5$ \\
\hline otal cholesterol, mg/dL & & $185.6 \pm 46.9$ & $187.5 \pm 46.0$ \\
\hline LDL-cholesterol, mg/dL & & $126.9 \pm 32.9$ & $127.1 \pm 43.0$ \\
\hline HDL-cholesterol, mg/dL & & $40.4 \pm 9.7$ & $36.8 \pm 12.5$ \\
\hline riglycerides, mg/dL & & $130.2 \pm 65.8$ & $133.6 \pm 71.2$ \\
\hline \multicolumn{4}{|l|}{ Treatment } \\
\hline spirin, (\%) & & 98.1 & 94.4 \\
\hline lopidogrel, (\%) & & 98.1 & 100.0 \\
\hline Beta-blockers, (\%) & & 60.4 & 61.1 \\
\hline tatins, $(\%)$ & & 100.0 & 100.0 \\
\hline Anti-coagulants, (\%) & & 100.0 & 100.0 \\
\hline ibrinolytics, (\%) & & 13.2 & 16.7 \\
\hline $\mathrm{PCI} \|,(\%)$ & & 5.7 & .6 \\
\hline
\end{tabular}

* Low neopterin group $<14.72 \mathrm{nmol} / \mathrm{L}$ $\dagger$ High neopterin group $\geq 14.72 \mathrm{mmol} / \mathrm{L}$ $\ddagger \mathrm{CAD}=$ Coronary artery disease $\S$ STEMI = ST-elevation myocardial infarction $\| \mathrm{PCl}=$ Percutaneous coronary intervention

Table 2. Type and proportions of CV events throughout the study in cohort of 71 ACS subjects

\begin{tabular}{lcc}
\hline \multicolumn{2}{c}{ Type of $\mathrm{CV}^{*}$ events } & Percentage (\%) \\
\hline Cardiovascular death & & 19.0 \\
Recurrent myocardial infarction & 9 & .5 \\
Recurrent myocardial ischemia & & 71.5 \\
Stroke $\quad 0$ & & .0 \\
\hline
\end{tabular}

${ }^{*} \mathrm{CV}=$ Cardiovascular 


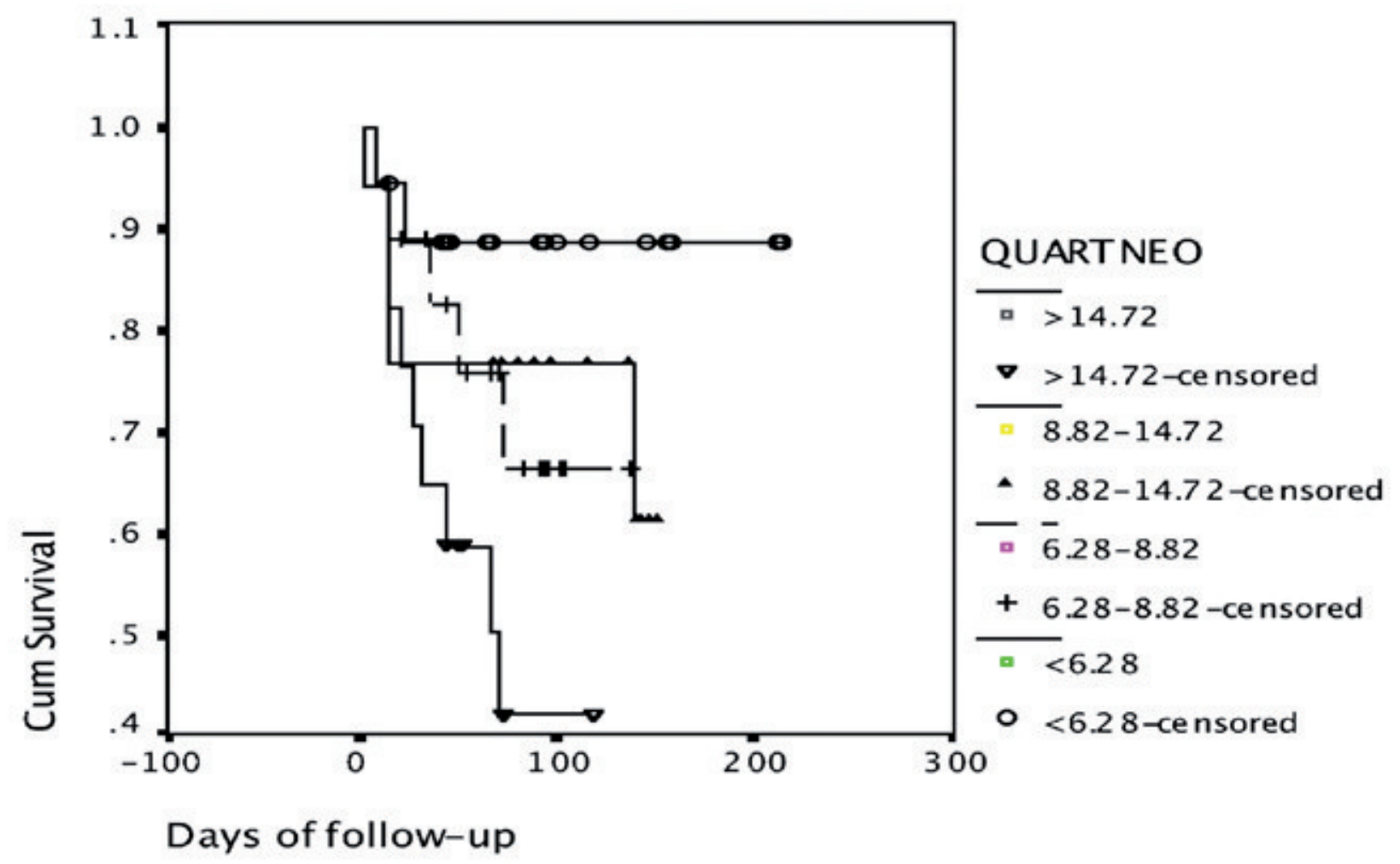

Figure 1. Estimates of $\mathrm{CV}$ events across quartiles of baseline neopterin. The log-rank test compared the difference in $\mathrm{CV}$ events across increasing quartiles of neopterin $(P=0.047)$.

Table 3. Relative risk of CV events Among Subjects With Highest Neopterin quartile group and Other Predictors in Multivariable Adjustment

\begin{tabular}{|c|c|c|c|c|c|}
\hline Predictors & & $\mathrm{RR}^{*}$ & & $95 \% \mathrm{Cl} \dagger$ & $P$ value \\
\hline Age & & .52 & 0 & $.60-3.84$ & 0.37 \\
\hline Hypertension & & .04 & 0 & $.38-2.83$ & 0.94 \\
\hline Diabetes mellitus 1 & & .17 & 0 & $.41-3.28$ & 0.76 \\
\hline Family history of CAD $\ddagger$ & 0 & .96 & 0 & $.36-2.54$ & 0.95 \\
\hline Neopterin (4th Q vs. 1st Q§) & & 5.84 & & $1.19-28.47$ & 0.029 \\
\hline
\end{tabular}

${ }^{*} \mathrm{RR}=$ relative risk

$+\mathrm{Cl}=$ confidence interval

$\mp C A D=$ coronary artery disease

$\S Q=$ quartile

This model adjusts for age, history of hypertension, diabetes mellitus and family history of CAD. 


\section{Discussion}

The results of our study show that neopterin, a biomarker of activated macrophage, predicts adverse $\mathrm{CV}$ events during follow-up in subjects with ACS. To our knowledge, this is the first clinical study performed in non-Caucasian population. After multivariable adjustment, patients in the highest quartile of serum neopterin concentrations had a 5 to 6-fold higher risk of developing adverse CV events than those in the lowest quartile. This association was present across a range of baseline characteristics and was statistically significant after adjustment for conventional risk factors, biochemical and treatment allocation. Thus, serum neopterin may be useful as prognostic factor in patients with ACS. Our results confirm and are consistent with previous findings from other study (12). This finding strongly suggests that increased activation of monocyte-macrophage system is a specific feature of high-risk ACS patient for CV death or recurrent acute coronary events.

Increased circulating T-lymphocyte activity, which leads to elevated production of interferon- $\gamma$, is implicated in the pathogenesis of ACS (17). CD4 ${ }^{+}$and CD8 ${ }^{+}$T-lymphocyte are proven to activate in patients with unstable angina and their activation state lasts 6 to 8 weeks (18). They are largely of the T-helper (Th1) subtype, which secretes interleukin-2, tumor necrosis factor- $\alpha$ and interferon- $\gamma$, which causes macrophage activation, vascular activation, and inflammation (19). Production of neopterin by activated macrophage is mainly in response to stimulation by interferon- $\gamma$ released by activated T-lymphocyte (19). Consequently, serum neopterin concentrations may represent the whole state of cell-mediated immunity within subjects with ACS.

Serum neopterin as a biomarker of macrophage activation is significantly increased in patients with AMI and unstable angina shortly after the onset of symptoms (after period of 72 hours) (20). Further, in regard to reliability of neopterin measurement, there is no significant 'sex dependence' and values for subjects younger or older than 75 years were significantly higher (21). This is the reason why we measured neopterin at $3^{\text {rd }}$ hospitalization day, and the age-range of our sample was only limited from 30 to 74 years old. The present study may be considered to be valid based on several reasons: a) the recruited samples represent the population of ACS in Bali, with spectrum ranged from unstable angina, NSTEMI and STEMI $(22), \mathrm{b})$ the follow-up was long enough for assessing the clinical outcomes, with 213 median-days, c) the clinical outcomes were assessed with no knowledge of the result of studied variable (neopterin), d) there were adjustments performed in order to prove the true association between neopterin concentration and the risk of $\mathrm{CV}$ events.

Recently, the prognostic value of elevated serum neopterin was studied in chronic stable angina pectoris (8). The cut-off point of neopterin to predict future adverse cardiac events in that study is lower than ours (7 nmol/L vs. $14.72 \mathrm{nmol} / \mathrm{L}$ ). This lower cut-off point is basically due to different studied population, namely chronic stable angina which represents less inflammatoric state than ACS population. Large-scale data on neopterin cut-off points in ACS actually are lacking. Ray et.al. (2007) determined neopterin cutoff point of $\geq 12.11 \mathrm{nmol} / \mathrm{L}$ in ACS population (12). Although the population is about the same as ours, but the cut-off point is even lower. Probably, our population is more inflammatoric, as represented by higher proportion of CV risk factors found (such as, diabetes mellitus, hypertension and LDL-cholesterol concentration). Percentage of STEMI in our study is more than Ray's study. Another reported study on 210 patients with NSTEMI from the FLORIDA (Fluvastatin On Risk Diminishing after Acute myocardial infarction) trial used a threshold of $9.7 \mathrm{nmol} / \mathrm{L}$, which represented the lower limit of the upper tertile. But it showed similar results to the present finding over a 1-year period of follow-up (23).

\section{LIMITATION OF THE STUDY}

The study limitation is the relatively small size of recruited subjects, though the study power is statistically modest. The neopterin cut-off point of $\geq 14.72 \mathrm{nmol} / \mathrm{L}$ used to define a higher level in the present study was not specified a priori and was based on the available neopterin data post hoc. Nevertheless, validation of this cut-off point in ACS population needs to be clarified in large prospective study. However, even with the relatively small sample size, elevated serum neopterin concentration was considered as a useful prognostic factor. 


\section{CONCLUSION}

Our study shows that elevated serum neopterin concentrations are associated with development of adverse CV events in ACS patients. This association is independent of the severity of ACS and baseline CV risk factors, as well as treatment allocation.

\section{Acknowledgments:}

We would like to express gratitude to Prof. Putu Sutisna, MD from the Faculty of Medicine, UDAYANA University for inspiration and helpful correction of this manuscript.

\section{References}

1. Ross R. The pathogenesis of atherosclerosis: a perspective for the 1990s. Nature. 1993; 362: $801-809$

2. Galis ZS, Sukhova GK, Lark MW, Libby P. Increased expression of matrix metalloproteinases and matrix degrading activity in vulnerable regions of human atherosclerotic plaques. $\mathrm{J}$ Clin Invest. 1994; 94: 2493 - 2503.

3. Hannson GK. Inflammation, Atherosclerosis, and Coronary Artery Disease. N Eng J Med. 2005; 352: 1685 - 1695.

4. Huber C, Batchelor JR, Fuchs D, Hausen A, Lang A, Niederwieser D, Reibnegger G, Swetly P, Troppmair J and Wachter H. Immune response-associated production of neopterin. Release from macrophages primarily under control of interferon-gamma. J Exp Med. 1984; 160: 310 - 316.

5. Werner-Felmayer G, Werner ER, Fuchs D, Hausen A, Reibnegger G, Schmidt K, Weiss G, Wachter H. Pteridine biosynthesis in human endothelial cells. Impact on nitric oxide-mediated formation of cyclic GMP. J Biol Chem. 1993; 268: 1842 -1846 .

6. Berdowska A, Zwirska-Korczala K. Neopterin measurement in clinical diagnosis. J Clin Pharm Ther. 2001; 26: 319 - 329.

7. Avanzas P, Arroyo-Espliguero R, Cosin-Sales J, Aldama G, Pizzi C, Quiles J, Kaski JC. Markers of inflammation and multiple complex stenoses (pancoronary plaque vulnerability) in patients with non-ST segment elevation acute coronary syndromes. Heart. 2004; 90: 847 - 852.

8. Avanzas P, Arroyo-Espliguero R, Quiles J, Roy D, Kaski JC. Elevated serum neopterin predicts future adverse cardiac events in patients with chronic stable angina pectoris. Eur Heart J. 2005; 26: 457 - 463.

9. Gupta S, Frederick S, Schwartzman RA, Holt DW, Kaski JC. Serum neopterin in acute coronary syndromes. Lancet. 1997; 349: 1252 - 1253.
10. Garcia-Moll X, Cole D, Zouridakis E, Kaski JC. Increased serum neopterin: a marker of coronary artery disease activity in women. Heart. 2000; 83: 346 - 350.

11. Avanzas P, Arroyo-Espliguero R, Cosin-Sales J, Quiles J, Zouridakis E, Kaski JC. Prognostic value of neopterin levels in treated patients with hypertension and chest pain but without obstructive coronary artery disease. Am J Cardiol. 2004; 93: 627 - 629 .

12. Ray KK, Morrow DA, Sabatine MC, Shui A, Rifai N, Cannon CP, Braunwald E. Long-Term Prognostic Value of Neopterin. A Novel Marker of Monocyte Activation in Patients With Acute Coronary Syndrome. Circulation. 2007; 115: 3071 - 3078.

13. Chobanian AV, Bakris GL, Black HR, Chusman WC, Green LA, Izzo, Jr. JL, Jones DW, Materson BJ, Oparil S, Wright, Jr JT, Rocella EJ. The seventh report of the Joint Committee on Prevention, Detection, Evaluation and Treatment of High Blood Pressure. JAMA. 2003; 289: 2560 - 2572.

14. American Diabetes Association. Standard of Medical care in Diabetes. Diabetes Care. 2006; 29: S4 - S42.

15. Al-Delaemy WK, Willet DC, Manson JE, Speizer FE, Hu FB. Smoking and Mortality Among Women with Type-2 Diabetes, Diabetes Care. 2001; 24(12): 2043 - 2048.

16. Westermann J, Thiemann F, Gerstner L, Tatzber F, Kozák I, Bertsch T, Krüger C. Evaluation of a New Simple and Rapid Enzym-Linked Immunosorbent Assay Kit for Neopterin Determination. Clin Chem Lab Med. 2000; 38(4): 345 $-353$.

17. Liuzzo G, Goronzy SL, Yang H, Kopecky SL, Holmes DR, Frye $\mathrm{RL}$, Weyand CM. Monoconal T-cell proliferation and plaque instability in acute coronary syndromes. Circulation. 2000; 101: $2883-2888$.

18. Serneri GGN, Prisco D, Martini F, Gori AM, Brunelli T, Poggesi L, Rostagno C, Gensini GF, Abbate R. Acute T-Cell activation Is Detectable in Unstable Angina. Circulation. 1997; 95: $1806-1812$.

19. Hansson GK. Inflammation, atherosclerosis, and coronary artery disease. N Eng J Med. 2005; 352: 1685 - 1695.

20. Auer J, Berent R, Labetanig E, Eber B. Serum neopterin and activity of coronary artery disease. Heart Dis. 2001; 3(5): $297-301$

21. Werner ER, Bichler A, Dexenbichler G, Fuche D, Fulth LC, Hausen A, Hetzel H, Relbneger G, Wachter H. Determination of Neopterin in Serum and Urine. Clin Chem. 1987; 33(1): $62-66$.

22. Santoso A, Wita W, Suastika K. Risk of Acute Myocardial Infarction was Associated with Hypoadiponectinemia. Folia Medica Indonesiana. 2006. vol. 42. Supplement No: 5 (241 - 246).

23. Van Haelst PL, Liem A, van Boven AJ, Veeger NJ, van Veldheuisen DJ, Gans RO, Ziljstra F. Usefulness of elevated neopterin and $\mathrm{C}$-reactive protein levels in predicting cardiovascular events in patients with non-Q wave myocardial infarction. Am J Cardiol. 2003; 92: 1201 - 1203. 